\title{
Cancer incidence and survival among Métis adults in Canada: results from the Canadian census follow-up cohort (1992-2009)
}

\author{
Maegan V. Mazereeuw MPH, Diana R. Withrow PhD, E. Diane Nishri MHSc, Michael Tjepkema MPH, \\ Eduardo Vides MD MPH, Loraine D. Marrett PhD
}

Cite as: CMAJ 2018 March 19;190:E320-6. doi: 10.1503/cmaj.170272

\begin{abstract}
BACKGROUND: Métis people are 1 of 3 Aboriginal groups recognized by the Canadian constitution. We estimated site-specific incidence rates and survival for the most common cancers among Métis adults in Canada and compared these with rates among non-Aboriginal adults in Canada.
\end{abstract}

METHODS: We examined responses to the 1991 long-form census, including selfreported Métis ancestry linked to national mortality and cancer databases for followup from 1992 to 2009. We estimated agestandardized incidence rates and 5-year relative survival. We determined relative risk (RR) of cancer among Métis and nonAboriginal adults using Poisson regression, and estimated excess mortality rate ratios using ethnicity-specific life tables.

RESULTS: For all cancers and both sexes combined, cancer incidence was similar for Métis and non-Aboriginal adults. However, incidence was significantly higher among Métis adults than among non-Aboriginal adults for the following cancers: female breast (RR 1.18, 95\% confidence interval $[\mathrm{Cl}] 1.02-1.37)$, lung (RR 1.34, 95\% Cl 1.18-1.52), liver (RR 2.09, $95 \%$ Cl 1.30-3.38), larynx (RR 1.60, 95\%
Cl 1.03-2.48), gallbladder (RR 2.35, 95\% Cl 1.12-4.96) and cervix (RR 1.84, 95\% $\mathrm{Cl}$ 1.23-2.76). Métis people had poorer survival for prostate cancer (excess mortality rate ratio $2.60,95 \% \mathrm{Cl} 1.52-4.46$ ).

INTERPRETATION: We found higher incidence for several cancers and poorer survival after prostate cancer among Métis adults. Several of these disparities may be related to lifestyle factors (including tobacco use, obesity and lack of cancer screening), providing evidence to support development of public health policy and health care to address cancer burden in the Métis people of Canada.
M étis people are 1 of the 3 distinct groups named in the Constitution Act of 1982 as the "aboriginal peoples of Canada," explicitly defined as "Indian [now referred to as 'First Nations'], Inuit, and Métis peoples of Canada." Aboriginal peoples in Canada experience greater poverty and unemployment, lower levels of education and higher prevalence of several known risk factors for chronic disease (tobacco smoking, obesity and unhealthy diet) relative to non-Aboriginal Canadians. ${ }^{2,3}$ These diverse influences and disadvantages have ultimately resulted in poorer health outcomes, such as lower life expectancy ${ }^{4,5}$ and higher prevalences of chronic conditions. ${ }^{3}$ The lack of racial or ethnic identifiers in Canadian health databases, including cancer registries, has limited our ability to accurately determine and effectively address priorities for chronic disease prevention in these populations. ${ }^{6}$

The Métis people of Canada are descendants of the offspring of early unions between First Nations women and European fur traders. Subsequent intermarriage of these mixed-ancestry individuals led to the genesis of a new Aboriginal people - the Métis people with their own unique culture, traditions, language (Michif), way of life, collective consciousness and nationhood (the Métis Nation). ${ }^{7}$ Métis constitute $1.4 \%$ of the Canadian population, numbering over 450000 individuals who are dispersed across the country, with particular concentrations in Ontario and the Prairie provinces. ${ }^{8}$ The paucity of formal national registration systems for Métis, coupled with the fact that Métis people tend not to cluster in specific geographic areas (unlike many Inuit and First Nation communities), has contributed to underrepresentation of Métis in the health literature relative to their share of the population. ${ }^{9}$ Furthermore, no single definition of Métis exists, which often makes comparisons between studies and generalization of results challenging. National data about cancer incidence among Métis are lacking, and Métis-specific cancer survival has never been estimated. Three provincial-level studies of cancer incidence among Métis in Canada had too few cases to produce precise estimates of differences between Métis people and the general population. ${ }^{10-12}$ 
We examined patterns of site-specific cancer incidence and survival among Métis people in Canada from 1992 to 2009 and evaluated their risk relative to the non-Aboriginal population.

\section{Methods}

\section{Study cohort and data sources}

The 1991 Canadian Census Health and Environment Cohort (previously known as the 1991 Census Mortality Cohort) is the largest population-based cohort in Canada and has been described in detail elsewhere. ${ }^{13,14}$ Briefly, to form the cohort, respondents to the 1991 long-form census aged 25 years or older on June 4, 1991 (census day), were probabilistically linked to nonfinancial tax summary files for the 1990 and 1991 tax years to add personal identifiers not stored with the census. Those found to have a tax record in one of these years were subsequently matched to the Canadian Mortality Database (1992-2009) and the Canadian Cancer Registry (1992-2009). ${ }^{13}$ The final cohort for this study was composed of 2.7 million individuals who represented a $15 \%$ sample of the Canadian population aged 25 or older on census day.

The Canadian Mortality Database is an administrative database maintained by Statistics Canada and containing demographic and medical (cause of death) information for all deaths from all provincial and territorial vital statistics registries. ${ }^{15}$

The Canadian Cancer Registry, an amalgamation of the 13 provincial and territorial cancer registries, includes all cancers diagnosed in Canadian residents since 1992. ${ }^{16}$ The component registries differ somewhat in their methods. In particular, the Quebec Tumour Registry has historically relied almost exclusively on hospital data, which results in a different definition of date of diagnosis from those in other jurisdictions, which generally have access to pathology reports. Because this definition affects the estimated time between diagnosis and death, especially for cancers with poor survival, ${ }^{17,18}$ we excluded Quebec residents from the survival analysis.

\section{Definitions}

\section{Diagnosis of cancer}

We counted multiple invasive cancers in the same individual according to the rules for multiple primary cancers of the International Agency for Research on Cancer. ${ }^{19}$ We grouped cancers according to the site recode for the International Classification of Diseases for Oncology (ICD-O-3) of the Surveillance, Epidemiology, and End Results Program (US National Cancer Institute) ${ }^{20}$ For the incidence analysis, we included individuals aged 25 to 99 years at diagnosis, and excluded those with a death date preceding the diagnosis date. We included all cancers with case counts of 5 or more in the Métis population. For the survival analysis, we included individuals aged 45 to 90 years at diagnosis (as described in the "Statistical analysis" section, below), and excluded cases registered solely on the basis of the death certificate or autopsy and those with death date preceding diagnosis date (which together accounted for less than $3 \%$ of Métis and of nonAboriginal cancer cases). To ensure a large enough sample size for stability in the estimates, we performed the survival analysis only for the 4 most common cancer types.

\section{Date of death}

We ascertained each person's date of death using a derived variable based on date or dates of death as reported in the Canadian Mortality Database, tax summary files and the Canadian Cancer Registry.

\section{Aboriginal ancestry}

We classified respondents as Métis or non-Aboriginal on the basis of answers to questions pertaining to Aboriginal ancestry in the 1991 long-form census. Respondents were asked to select the cultural group or groups to which their ancestors belonged, with no limit on the number of ancestries that could be reported. We classified a person as Métis if they reported 2 or fewer ancestries, one of which was Métis. We excluded from the Métis category individuals who reported registration under the Indian Act or membership in an Indian Band or First Nation (or both), given the distinct nationhood of Métis people. No gold standard identifier exists. In later censuses, questions have asked about both Aboriginal identity and ancestry. The definition we chose was informed by comparisons between responses to the identity and ancestry concepts in later censuses, whereby most of the individuals meeting the above-described definition of Métis based primarily on ancestry also reported Métis identity.

Respondents were considered to be of non-Aboriginal ancestry if they were not First Nations (North American Indian ancestry only, registration under the Indian Act, or membership in an Indian Band or First Nation), Métis (as defined previously) or Inuit (Inuit ancestry only).

\section{Income, rurality and region}

Statistics Canada constructed area-level income adequacy quintiles using the ratio of total income from all sources combined across all members of an economic family unit to low-income cut-offs from the 1991 Census Dictionary. ${ }^{21}$ Rurality was a dichotomous area-level variable defined by Statistics Canada as the population living in towns and municipalities outside the commuting zone of larger urban centres (i.e., the non-census metropolitan area/non-census agglomeration population). ${ }^{22}$ Region was determined according to the province or territory of residence on census day.

\section{Statistical analysis}

\section{Incidence of cancer}

Person-time was accumulated from Jan. 1, 1992. For all cancers combined, follow-up ended at the earliest of date of death or Dec. 31,2009 . For site-specific cancer incidence, follow-up was censored at the date of diagnosis of that cancer if a cancer was diagnosed. Incidence rates were age-standardized using the World Standard Population. ${ }^{23}$ We performed a subgroup analysis to evaluate patterns of site-specific cancer incidence by region of residence.

We estimated relative risk (RR) comparing the rate of cancer in Métis and non-Aboriginal adults using 2 Poisson models, the 
first controlling for age and sex, and the second additionally controlling for income and rurality. All incidence analyses were performed using SAS version 9.4.

\section{Survival}

We estimated relative survival using ethnicity-, age-, sex- and calendar time-specific life tables created from the cohort at large using flexible parametric models implemented with the stpm2 command in Stata version 13 (StataCorp LP). We restricted the life tables to ages 45 to 90 because within this age span, mortality rates in the census cohort were more stable for Métis respondents. Stable underlying or expected mortality rates are important for measuring relative survival because relative survival is a ratio of the observed survival among patients with cancer to the expected survival of members of the general population matched for ethnicity, age, sex and calendar period.

We produced 5 -year excess mortality rate ratios with 2 flexible parametric survival models, the first controlling for age, sex and decade of diagnosis (1992-2000 v. 2001-2009), and the second additionally controlling for income and rurality. The 5-year relative survival ratios were age-standardized to International Cancer Survival Standards. ${ }^{24}$ We restricted these age-standardized survival ratios to cases diagnosed from 2001 to 2009 because although excess mortality rate ratios tended not to change significantly over time, survival generally improved over time.

\section{Ethics approval}

The study was approved by the research ethics board of the University of Toronto (protocol 29323).

\section{Results}

Among the 11050 adults aged 25 to 99 years with Métis ancestry, 1090 cancers were diagnosed over about 185000 person-years. Relative to non-Aboriginal adults, Métis adults were significantly younger, more likely to reside in rural areas and the Prairie provinces (Alberta, Saskatchewan and Manitoba) and to have lower levels of income and education (Table 1).

For all cancers and both sexes combined, cancer incidence was similar for Métis and non-Aboriginal adults (Table 2). Cancer incidence was similar for Métis men and significantly higher for Métis women relative to their non-Aboriginal counterparts. Métis adults had significantly higher incidence of cancers of the lung (men and women), female breast and cervix, as well as liver, larynx and gallbladder (both sexes combined), relative to their nonAboriginal peers, whereas the risk was significantly lower for colorectal cancers in women and for melanoma and leukemia in both sexes combined. The incidence of cancers of the kidney, stomach and ovary were numerically higher among Métis than among non-Aboriginal adults, but these differences were not statistically significant. In the subgroup analysis, lung was the only cancer site for which some variation was observed by region, with the greatest disparity between Métis and non-Aboriginal adults in the Ontario/Quebec region (Figure 1).

The excess mortality rate ratio comparing survival among Métis with that among non-Aboriginal adults was numerically lower for
Table 1: Demographic characteristics of cohort members

\begin{tabular}{|c|c|c|}
\hline \multirow[b]{2}{*}{ Characteristic } & \multicolumn{2}{|c|}{$\begin{array}{l}\text { Ethnic group; } \\
\text { no. }(\%) \text { of respondents* }\end{array}$} \\
\hline & $\begin{array}{c}\text { Non-Aboriginal } \\
n=2652770\end{array}$ & $\begin{array}{c}\text { Métis } \\
n=11050\end{array}$ \\
\hline \multicolumn{3}{|l|}{ Sex } \\
\hline Women & 1332225 (50.2) & 5505 (49.8) \\
\hline Men & 1320545 (49.8) & $5545(50.2)$ \\
\hline \multicolumn{3}{|c|}{ Age at cohort entry, yr } \\
\hline $25-34$ & $738335(27.8)$ & 4405 (39.9) \\
\hline $35-44$ & $695985(26.2)$ & $3110(28.1)$ \\
\hline $45-54$ & $456715(17.2)$ & $1740(15.7)$ \\
\hline $55-64$ & $344905(13.0)$ & $1020(9.2)$ \\
\hline $65-74$ & $268165(10.1)$ & $515(4.7)$ \\
\hline $75-99$ & $148665(5.6)$ & $260(2.4)$ \\
\hline \multicolumn{3}{|c|}{ Region of residence $\dagger$} \\
\hline North & $15080(0.6)$ & $785(7.1)$ \\
\hline British Columbia & 315290 (11.9) & $665(6.0)$ \\
\hline Alberta & 236200 (8.9) & $2595(23.5)$ \\
\hline Saskatchewan & $92580(3.5)$ & $2330(21.1)$ \\
\hline Manitoba & $105085(4.0)$ & $2605(23.6)$ \\
\hline Ontario & $982175(37.0)$ & $835(7.6)$ \\
\hline Quebec & 687485 (25.9) & $1005(9.1)$ \\
\hline Atlantic & 218875 (8.3) & $230(2.1)$ \\
\hline
\end{tabular}

\section{Highest level of schooling}

No high school

907200 (34.2)

5885 (53.3)

High school, with or without

trades certificate

968885 (36.5)

3645 (33.0)

Postsecondary

413065 (15.6)

$1150(10.4)$

(non-university)

University degree

$363620(13.7)$

$370(3.3)$

\section{Income quintile}

1 (lowest)

439965 (16.6)

3425 (31.0)

2

3

4

510690 (19.3)

$2540(23.0)$

550505 (20.8)

2100 (19.0)

570155 (21.5)

1815 (16.4)

5 (highest)

$581450(21.9)$

$1170(10.6)$

\section{Rurality}

Rural

600715 (22.6)

5995 (54.3)

Urban

2052055 (77.4)

*According to Statistics Canada protocols, the counts in this table, including totals, are randomly rounded either up or down to a multiple of 5 . As such, when data are summed or grouped, the total value may not match the individual values because totals and subtotals are rounded independently. Similarly, percentages are calculated from the rounded data, so those for a given variable may not sum to $100 \%$.

†"North" consists of Yukon, Northwest Territories and Nunavut; "Atlantic" consists of New Brunswick, Nova Scotia, Prince Edward Island, and Newfoundland and Labrador. 
Table 2: Age-standardized cancer incidence and relative risk of cancer among Métis and non-Aboriginal adults, 1992-2009, by sex* and cancer site, Canada

\begin{tabular}{|c|c|c|c|c|c|}
\hline \multirow[b]{2}{*}{ Cancer site } & \multirow{2}{*}{$\begin{array}{l}\text { No. of cases } \\
\text { in Métis } †\end{array}$} & \multicolumn{2}{|c|}{ Ethnic group; ASR $¥$ per 100000} & \multicolumn{2}{|c|}{ Model no.; RR (95\% CI) } \\
\hline & & Non-Aboriginal & Métis & Model $1 \S$ & Model 2ฯ \\
\hline All sites & 1090 & 514.3 & 546.4 & $1.06(1.00-1.12)$ & $1.05(0.99-1.11)$ \\
\hline Men & 570 & 560.7 & 558.2 & $0.99(0.91-1.07)$ & $0.97(0.89-1.06)$ \\
\hline Women & 525 & 457.4 & 533.0 & $1.14(1.04-1.24)$ & $1.13(1.04-1.24)$ \\
\hline Lung** & 225 & 73.3 & 114.0 & $1.50(1.32-1.70)$ & $1.34(1.18-1.52)$ \\
\hline Men & 125 & 89.8 & 125.8 & $1.39(1.18-1.64)$ & $1.22(1.03-1.43)$ \\
\hline Women & 95 & 57.5 & 107.8 & $1.67(1.38-2.03)$ & $1.56(1.28-1.89)$ \\
\hline Female breast & 165 & 146.0 & 165.2 & $1.12(0.97-1.30)$ & $1.18(1.02-1.37)$ \\
\hline Prostate & 145 & 157.3 & 150.2 & $0.96(0.82-1.11)$ & $1.00(0.86-1.17)$ \\
\hline Colorectal & 115 & 63.9 & 56.4 & $0.90(0.76-1.08)$ & $0.90(0.75-1.06)$ \\
\hline Men & 75 & 75.4 & 74.3 & $1.02(0.83-1.26)$ & $1.03(0.83-1.26)$ \\
\hline Women & 35 & 50.3 & 37.0 & $0.71(0.52-0.97)$ & $0.69(0.50-0.95)$ \\
\hline Non-Hodgkin lymphoma & 40 & 26.1 & 21.0 & $0.85(0.65-1.12)$ & $0.86(0.66-1.13)$ \\
\hline Kidney & 35 & 13.9 & 17.5 & $1.26(0.93-1.71)$ & $1.23(0.91-1.67)$ \\
\hline Stomach & 30 & 10.8 & 15.5 & $1.44(1.04-2.01)$ & $1.37(0.98-1.90)$ \\
\hline Bladder & 30 & 16.9 & 14.1 & $0.80(0.57-1.11)$ & $0.79(0.56-1.10)$ \\
\hline Oral and pharynx & 25 & 11.9 & 12.9 & $0.98(0.68-1.41)$ & $0.88(0.61-1.27)$ \\
\hline Pancreas & 25 & 11.6 & 12.2 & $1.04(0.71-1.53)$ & $1.03(0.70-1.51)$ \\
\hline Uterus & 25 & 28.4 & 25.7 & $0.89(0.62-1.28)$ & $0.91(0.64-1.32)$ \\
\hline Cervix & 25 & 11.0 & 22.9 & $2.05(1.37-3.06)$ & $1.84(1.23-2.76)$ \\
\hline Ovary & 25 & 16.5 & 22.6 & $1.38(0.94-2.03)$ & $1.41(0.96-2.08)$ \\
\hline Liver & 15 & 3.7 & 8.2 & $2.01(1.25-3.25)$ & $2.09(1.30-3.38)$ \\
\hline Larynx & 15 & 4.2 & 8.1 & $1.82(1.17-2.83)$ & $1.60(1.03-2.48)$ \\
\hline Thyroid & 15 & 10.3 & 6.6 & $0.69(0.42-1.13)$ & $0.76(0.47-1.24)$ \\
\hline Melanoma & 10 & 16.8 & 6.5 & $0.49(0.32-0.76)$ & $0.54(0.35-0.84)$ \\
\hline Brain and CNS & 10 & 8.5 & 4.6 & $0.55(0.30-1.03)$ & $0.57(0.31-1.06)$ \\
\hline Leukemia & 5 & 7.4 & 3.2 & $0.60(0.34-0.96)$ & $0.59(0.37-0.94)$ \\
\hline Gallbladder & 5 & 1.3 & 2.8 & $2.50(1.19-5.27)$ & $2.35(1.12-4.96)$ \\
\hline \multicolumn{6}{|c|}{$\begin{array}{l}\text { Note: ASR = age-standardized rate, } \mathrm{Cl}=\text { confidence interval, } C N S=\text { central nervous system, RR = relative risk. } \\
\text { *Breakdown by sex for all sites and the top } 4 \text { incident cancer sites (lung, female breast, prostate, colorectal). } \\
\text { †According to Statistics Canada protocols, the counts in this table, including totals, are randomly rounded either up or down to a multiple of } 5 \text {. When data are summed or grouped, th } \\
\text { total value may not match the individual values because totals and subtotals are rounded independently. } \\
\text { IStandardized to the World Standard Population. }{ }^{23} \\
\text { \$With adjustment for age and sex (if applicable) only. }\end{array}$} \\
\hline
\end{tabular}

breast (female), colorectal and prostate cancers, but the difference was statistically significant only for prostate cancer (Table 3).

\section{Interpretation}

In this study, for all cancers and both sexes combined, cancer incidence was not significantly different between Métis and nonAboriginal adults; all-cancer incidence also did not differ between Métis and non-Aboriginal men. However, cancer inci- dence was significantly higher for Métis women than for nonAboriginal women. Métis had higher incidence rates for lung cancer in men and women separately; for larynx, liver and gallbladder cancers in both sexes combined; and for certain cancers of the female reproductive system (breast, cervix); the incidence rate was numerically higher for ovary, but this result was not statistically significant. Conversely, Métis adults had lower incidence of colorectal cancer (women only) and of melanoma and leukemia for both sexes combined. 
Recent evidence shows the prevalence of cigarette smoking and obesity among Métis is high. ${ }^{2,25}$ These 2 factors are strongly associated with an increased risk of several cancer types ${ }^{26-28}$ and may explain, in part, the high incidence of lung, breast and certain other cancers in Métis adults. The higher incidence of cervical cancer in Métis women may be related to low screening participation, though limited evidence in more recent years suggests cervical screening participation rates are similar between Métis and non-Aboriginal women. ${ }^{2,29}$ Survival was numerically lower for Métis people with breast, colorectal and prostate cancer, but the

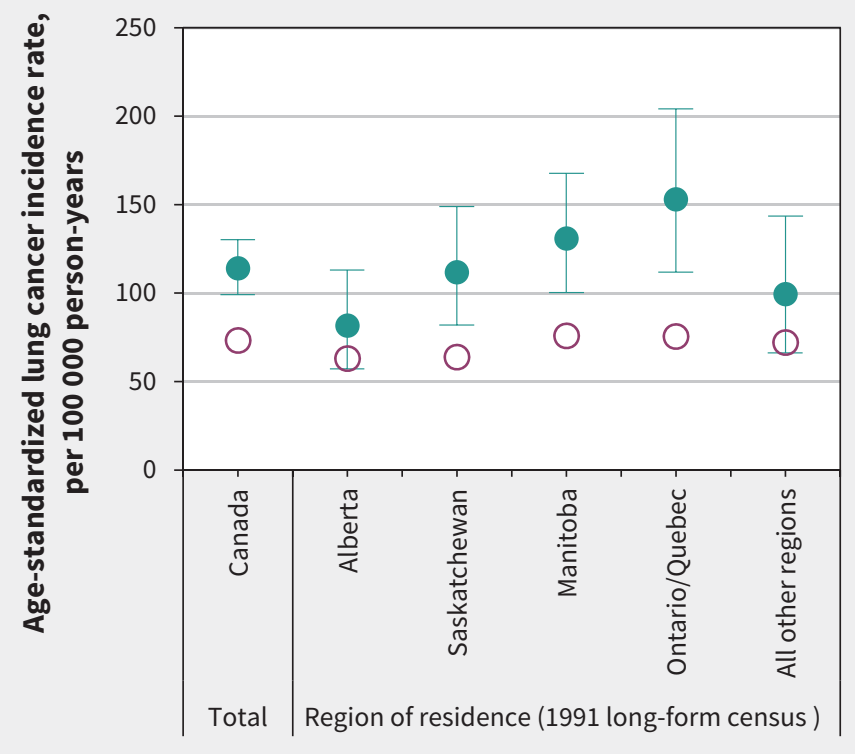

- Métis o non-Aboriginal

Figure 1: Age-standardized incidence rates for lung cancer among Métis and non-Aboriginal adults, by region of residence, both sexes combined, 1992-2009. Lung cancer = cancers of the lung and bronchus. Error bars indicate 95\% confidence intervals for Métis adults. Error bars for nonAboriginal adults were narrower than the data points in all regions and were therefore omitted for clarity of presentation.

\section{Table 3: Age-standardized 5-year relative survival by sex and ethnicity, and excess mortality rate ratios for Métis and} non-Aboriginal adults aged 45-90 at diagnosis, Canada excluding Quebec

\section{Sex and ethnic group; age-standardized 5-yr relative survival, \% $(95 \% \mathrm{Cl}) \neq \S$}

\begin{tabular}{|c|c|c|c|c|c|c|c|}
\hline \multirow{2}{*}{$\begin{array}{l}\text { Cancer } \\
\text { site }\end{array}$} & \multirow{2}{*}{$\begin{array}{l}\text { No. of } \\
\text { cancers in } \\
\text { Métis* } †\end{array}$} & \multicolumn{2}{|c|}{ Women } & \multicolumn{2}{|c|}{ Men } & \multicolumn{2}{|c|}{$\operatorname{EMRR}(95 \% \mathrm{Cl})^{*}$} \\
\hline & & Non-Aboriginal & Métis & Non-Aboriginal & Métis & Model 19 & Model $2^{\star \star}$ \\
\hline Lungt† & 190 & $20.1(19.1-21.1)$ & $15.0(10.0-22.6)$ & $12.8(12.1-13.6)$ & $9.1(5.4-15.2)$ & $1.03(0.88-1.22)$ & $1.00(0.85-1.18)$ \\
\hline Prostate & 135 & NA & NA & $93.2(92.5-93.9)$ & $78.2(65.4-93.4)$ & $3.58(2.04-6.30)$ & $2.60(1.52-4.46)$ \\
\hline Breast & 130 & $87.0(86.0-88.0)$ & $79.6(68.0-93.2)$ & NA & NA & $1.61(0.99-2.62)$ & $1.43(0.88-2.34)$ \\
\hline Colorectal & 90 & $62.6(61.2-64.0)$ & $56.3(43.2-73.4)$ & $64.1(62.9-65.4)$ & $55.1(65.4-93.4)$ & $1.14(0.79-1.64)$ & $1.14(0.79-1.64)$ \\
\hline
\end{tabular}

Note: $\mathrm{Cl}=$ confidence interval, $\mathrm{EMRR}=$ excess mortality rate ratio, $\mathrm{NA}=$ not applicable.

*Diagnosis between Jan. 1, 1992, and Dec. 31, 2009.

†According to Statistics Canada protocols, the counts in this table, including totals, are randomly rounded either up or down to a multiple of 5 . When data are summed or grouped, the total value may not match the individual values because totals and subtotals are rounded independently.

‡Diagnosis between Jan. 1, 2001, and Dec. 31, 2009.

$\S$ Age-standardized to International Cancer Survival Standards..$^{24}$

IAdjusted for age, sex (if applicable) and time period.

${ }^{\star \star}$ Adjusted for age, sex (if applicable), time period, income quintile and rurality.

t†Lung cancer $=$ cancers of the lung and bronchus. 
result was statistically significant only for prostate cancer. Controlling for income and rurality did little to explain the disparities in incidence or survival between the populations.

Only 3 studies, all at the provincial level, have previously examined cancer incidence in the Métis population. ${ }^{10-12}$ In Ontario, researchers found no difference between Métis and all Ontarians, except for a suggestion of lower overall incidence among Métis participants; however, the numbers were too small to be informative ( $n=168$ cancers among Métis participants during 2005-2007). ${ }^{10}$ The Manitoba study was larger $(n=2551$ cancers among Métis participants during 1998-2007) and, as in our study, showed similar incidence among Métis and all Manitobans for all sites combined and for colorectal and prostate cancers, with higher rates of lung cancer for Métis males and females. ${ }^{11}$ Unlike our results, Manitoba Métis women did not experience significantly higher rates of breast or cervical cancer. In Alberta ( $n=444$ cancers among Métis participants during 2007-2012), researchers reported results that were qualitatively similar to ours for all cancers combined and for lung, breast, prostate and colorectal cancers, although statistical significance was lacking except for lung cancer (both sexes combined and males), for which rates were significantly higher for Métis than for non-Métis Albertans. ${ }^{12}$ Finally, Tjepkema and colleagues ${ }^{4}$ looked at mortality among Métis people in Canada using the 1991 census cohort followed through 2001. As in our study of cancer incidence, those authors found similar all-cancer mortality rates in men but a higher rate in Métis women, higher lung cancer mortality in Métis women and similar colorectal cancer mortality rates for Métis people of both sexes, relative to nonAboriginal Canadians. Whereas we found elevated lung cancer incidence among Métis men, Tjepkema and colleagues ${ }^{4}$ did not report higher lung cancer-specific mortality.

\section{Strengths and limitations}

This study had both strengths and limitations. Using census data allowed for the identification of Métis according to self-reported ancestry, which is preferable to ethnic identifiers derived from the medical record, given that the latter are often based on subjective appraisal. ${ }^{30-32}$ However, the ideal classification of Métis would be based on self-identity, which was not requested in the 1991 census. The generalizability of data from Métis participants as defined in this cohort to the Métis people of today is unclear. The estimated size of the Métis population has increased since 1991 because of both natural growth and changes in the likelihood of reporting Métis identity on the census. ${ }^{33}$

Although our cohort began in 1991, it provides important information for planning and prevention efforts. A more recent cohort would not have allowed sufficient power to measure cancer burden among Métis participants. Our cohort had sufficient follow-up, with more than 18 years of combined data; however, we were still limited by a relatively small sample, which was too small to explore trends over time. Our data sources did not include stage of diagnosis or lifestyle risk factors, and we were consequently unable to explore the role of these factors in the observed disparities. Income was measured at the aggregate (rather than individual) level, and rurality was dichotomized, not taking into account potential differences between rural and remote areas. As a result, we cannot definitively conclude the absence of residual confounding by these factors.

\section{Conclusion}

The value of this work is in providing evidence to support public health policy and programming that can ultimately contribute to a reduction in the risk and burden of cancer and other chronic diseases among the Métis people of Canada. System-level efforts, with culturally appropriate risk-reduction strategies and emphasizing tobacco prevention and cessation and healthy weights, could be considered to reduce the high burden of potentially avoidable cancers. Furthermore, the higher incidence of cervical cancer among Métis women suggests that improvements in screening would yield benefits. The poorer survival of Métis patients with prostate cancer requires further research to identify the causes of the disparity and, where appropriate, potential actions to reduce them. Our limited understanding of cancer risk and burden in Aboriginal populations in Canada stems from a lack of ethnic identifiers in Canadian health databases. The development of more comprehensive information systems that include ethnic identifiers will be crucial to inform and evaluate strategies to reduce health disparities between Métis and the rest of the Canadian population.

\section{References}

1. Part II (section 35): Rights of the aboriginal peoples of Canada. In: Constitution Act, 1982, being Schedule B to the Canada Act 1982, 1982, c. 11 (UK).

2. Withrow DR, Amartey A, Marrett LD. Cancer risk factors and screening in the off-reserve First Nations, Métis and non-Aboriginal populations of Ontario. Chronic Dis Inj Can 2014;34:103-12.

3. Health at a glance: select health indicators of First Nations people living off-reserve, Métis and Inuit, 2007-2010. Ottawa: Statistics Canada; 2013. Cat no 82-624-X.

4. Tjepkema M, Wilkins R, Senécal S, et al. Mortality of Métis and registered Indian adults in Canada: an 11-year follow-up study. Health Rep 2009;20(4):31-51.

5. Tjepkema M, Wilkins R. Remaining life expectancy at age 25 and probability of survival to age 75 , by socio-economic status and Aboriginal ancestry. Health Rep 2011;22(4):31-6.

6. Minore B, Katt M, Hill ME. Planning without facts: Ontario's Aboriginal health information challenge. J Agromedicine 2009;14:90-6.

7. The Métis Nation. Ottawa: Métis National Council. Available: www.metisnation. ca/index.php/who-are-the-metis (accessed 2017 May 21).

8. Aboriginal peoples in Canada: First Nations people, Métis and Inuit. Ottawa: Statistics Canada; 2016. Available: Cat no 99-011-X. https://www12.statcan.gc.ca/ nhs-enm/2011/as-sa/99-011-x/99-011-x2011001-eng.cfm (accessed 2016 Mar. 3).

9. Young TK. Review of research on aboriginal populations in Canada: relevance to their health needs. BMJ 2003;327:419-22.

10. Withrow D, Kewayosh A, Marrett L. Cancer in the Métis Nation of Ontario: clinical significance report. Ottawa: Métis Nation of Ontario; 2012.

11. Bartlett JG, Sanguins J, Carter S, et al. Cancer and related health care utilization in the Manitoba Metis population. Winnipeg: Manitoba Metis Federation; 2011.

12. Sanchez-Ramirez DC, Colquhoun A, Parker S, et al. Cancer incidence and mortality among the Metis population of Alberta, Canada. Int J Circumpolar Health 2016;75:30059.

13. Wilkins R, Tjepkema M, Mustard C, et al. The Canadian census mortality followup study, 1991 through 2001. Health Rep 2008;19(3):25-43.

14. Peters PA, Tjepkema M, Wilkins R, et al. Data resource profile: 1991 Canadian census cohort. Int J Epidemiol 2013;42:1319-26.

15. Canadian Mortality Database. In: Microdata linkage program. Ottawa: Statistics Canada; 2017. Available: www.statcan.gc.ca/eng/health/link\#cmdb (accessed 2018 Feb. 6).

16. Canadian Cancer Registry (CCR). Ottawa: Statistics Canada; 2016. Available: www23.statcan.gc.ca/imdb/p2SV.pl?Function=getSurvey\&SDDS=3207 (accessed 2016 Dec. 28). 
17. Canadian cancer statistics 2014 - special topic: skin cancers. Toronto: Canadian Cancer Society; 2014.

18. Ellison LF, Gibbons L; Canadian Cancer Survival Analysis Group. Five-year relative survival from prostate, breast, colorectal and lung cancer. Health Rep 2001; 13(1):23-34.

19. Working Group Report. International rules for multiple primary cancers (ICD-0 third edition). Eur J Cancer Prev 2005;14:307-8.

20. Site recode ICD-O-3 (1/27/2003) definition. Bethesda (MD): National Cancer Institute; 2003. Available: http://seer.cancer.gov/siterecode/icdo3_d01272003 (accessed 2015 July 27).

21. 1991 census dictionary. Ottawa: Statistics Canada; 1992. Cat no 92-301E.

22. du Plessis V, Beshiri R, Bollman RD. Definitions of rural. Rural Small Town Can Anal Bull 2001;3(3):1-17. Statistics Canada Cat no 21-006-XIE.

23. Chapter 7: Age standardization. In: Parkin DM, Muir CS, Whelan SL, et al., editors. Cancer incidence in five continents. Vol 6. IARC Sci Publ 120. Lyon (France): International Agency for Research on Cancer; 1992:112-115.

24. Corazziari I, Quinn M, Capocaccia R. Standard cancer patient population for age standardising survival ratios. Eur J Cancer 2004;40:2307-16.

25. Gionet L, Roshanafshar S. Select health indicators of First Nations people living off reserve, Métis and Inuit. Ottawa: Statistics Canada; 2013. Cat no 82-624-X.
26. Parkin DM, Boyd L, Walker LC. 16. The fraction of cancer attributable to lifestyle and environmental factors in the UK in 2010. Br J Cancer 2011;105(Suppl 2):S77-81.

27. World Cancer Research Fund/American Institute for Cancer Research. Food, nutrition, physical activity and the prevention of cancer: a global perspective. Washington: American Institute for Cancer Research; 2007.

28. Cogliano VJ, Baan R, Straif K, et al. Preventable exposures associated with human cancers. J Natl Cancer Inst 2011;103:1827-39.

29. Martens P, Bartlett J, Burland E, et al. Profile of Metis health status and healthcare utilization in Manitoba: a population-based study. Winnipeg: Manitoba Centre for Health Policy; 2010.

30. Gomez SL, Le GM, West DW, et al. Hospital policy and practice regarding the collection of data on race, ethnicity, and birthplace. Am J Public Health 2003;93:1685-8.

31. Robertson H, Lumley J, Berg S. How midwives identify women as aboriginal or Torres Strait Islanders. Aust Coll Midwives Inc J 1995;8(3):26-9.

32. Clegg LX, Li FP, Hankey BF, et al. Cancer survival among US whites and minorities: a SEER (Surveillance, Epidemiology, and End Results) Program populationbased study. Arch Intern Med 2002;162:1985-93.

33. Guimond E, Kerr D, Beaujot R. Charting the growth of Canada's Aboriginal populations: problems, options and implications. Can Stud Popul 2004;31:55-82.

\section{Competing interests: None declared.}

This article has been peer reviewed.

Affiliations: Prevention and Cancer Control (Mazereeuw, Withrow, Nishri, Marrett), Cancer Care Ontario; Dalla Lana School of Public Health (Withrow, Marrett), University of Toronto, Toronto, Ont.; Health Analysis Division (Tjepkema), Statistics Canada; Métis National Council (Vides), Ottawa, Ont.

Contributors: Diana Withrow, Michael Tjepkema and Loraine Marrett conceptualized and implemented the study. Maegan Mazereeuw and Diana Withrow performed the data analysis. Diane Nishri, Michael Tjepkema and Loraine Marrett provided methodologic and subject matter expertise and guided the statistical analysis. Eduardo Vides provided content expertise and data interpretation from the perspective of the Métis Nation. Maegan Mazereeuw drafted the article, and all of the authors critically reviewed it for important intellectual content. All of the authors gave final approval of the version to be published and agreed to be accountable for all aspects of the work.

Funding: The work was supported by a Canadian Institutes of Health Research Open Operating Grant entitled "Cancer incidence and survival in First Nations and Métis adults in Canada: follow-up of the 1991 census cohort."

Accepted: Sept. 12, 2017

Correspondence to: Maegan Mazereeuw, mmazereeuw@cihi.ca 